$1-2-2017$

\title{
A Light-Weight Opportunistic Forwarding Protocol with Optimized Preamble Length for Low-Duty-Cycle Wireless Sensor Networks
}

Hai-Ming Chen

chenhaiming@ict.ac.cn

Li Cui

Icui@ict.ac.cn

Gang Zhou

College of William and Mary, gzhou@cs.wm.edu

Follow this and additional works at: https://scholarworks.wm.edu/aspubs

\section{Recommended Citation}

Chen, Hai-Ming; Cui, Li; and Zhou, Gang, A Light-Weight Opportunistic Forwarding Protocol with Optimized Preamble Length for Low-Duty-Cycle Wireless Sensor Networks (2017).

$10.1007 / \mathrm{s} 11390-017-1712-4$

This Article is brought to you for free and open access by the Arts and Sciences at W\&M ScholarWorks. It has been accepted for inclusion in Arts \& Sciences Articles by an authorized administrator of W\&M ScholarWorks. For more information, please contact scholarworks@wm.edu. 
Chen HM, Cui L, Zhou G. A light-weight opportunistic forwarding protocol with optimized preamble length for low-dutycycle wireless sensor networks. JOURNAL OF COMPUTER SCIENCE AND TECHNOLOGY 32(1): 168-180 Jan. 2017. DOI 10.1007/s11390-017-1712-4

\title{
A Light-Weight Opportunistic Forwarding Protocol with Optimized Preamble Length for Low-Duty-Cycle Wireless Sensor Networks
}

\author{
Hai-Ming Chen ${ }^{1}$, Member, CCF, ACM, IEEE, Li Cui ${ }^{1}$, Senior Member, CCF \\ and Gang Zhou ${ }^{2}$, Senior Member, ACM, IEEE \\ ${ }^{1}$ Institute of Computing Technology, Chinese Academy of Sciences, Beijing 100190, China \\ ${ }^{2}$ Department of Computer Science, College of William and Mary, Williamsburg, VA 23185, U.S.A. \\ E-mail: \{chenhaiming, lcui\}@ict.ac.cn; gzhou@cs.wm.edu
}

Received February 25, 2016; revised September 5, 2016.

\begin{abstract}
In wireless sensor networks, sensed information is expected to be reliably and timely delivered to a sink in an ad-hoc way. However, it is challenging to achieve this goal because of the highly dynamic topology induced from asynchronous duty cycles and temporally and spatially varying link quality among nodes. Currently some opportunistic forwarding protocols have been proposed to address the challenge. However, they involve complicated mechanisms to determine the best forwarder at each hop, which incurs heavy overheads for the resource-constrained nodes. In this paper, we propose a light-weight opportunistic forwarding (LWOF) scheme. Different from other recently proposed opportunistic forwarding schemes, LWOF employs neither historical network information nor a contention process to select a forwarder prior to data transmissions. It confines forwarding candidates to an optimized area, and takes advantage of the preamble in low-power-listening (LPL) MAC protocols and dual-channel communication to forward a packet to a unique downstream node towards the sink with a high probability, without making a forwarding decision prior to data transmission. Under LWOF, we optimize LPL MAC protocol to have a shortened preamble (LWMAC), based on a theoretical analysis on the relationship among preamble length, delivery probability at each hop, node density and sleep duration. Simulation results show that LWOF, along with LWMAC, can achieve relatively good performance in terms of delivery reliability and latency, as a receiver-based opportunistic forwarding protocol, while reducing energy consumption per packet by at least twice.
\end{abstract}

Keywords wireless sensor network, low duty cycle, low power listening, opportunistic forwarding

\section{Introduction}

Wireless sensor networks (WSNs) are often deployed to collect physical information, such as environmental temperature, water quality, and target location, periodically or when interested events occur. Sensed information is expected to be reliably and timely forwarded to a sink in an ad-hoc way. However, it is observed that data transmissions among sensor nodes are lossy ${ }^{[1]}$, and prone to being interfered with each other and other networks sharing the same $2.4 \mathrm{GHz}$ ISM band ${ }^{[2]}$. The lossy links and dynamic interferences make it challenging for sensor nodes to reliably and timely deliver data to the $\operatorname{sink}^{[3]}$. Furthermore, sensor nodes usually work with sleep-wakeup schedules to save power, and the asynchronous duty cycles among nodes aggravate packet loss and transmission delay ${ }^{[4]}$.

Currently some data collection protocols aware of dynamic link quality, such as CTP (Collection Tree Protocol $)^{[5]}$, have been designed specifically for WSNs. In addition, some duty cycle synchronization mechanisms, such as SCP (Scheduled Channel Probing) ${ }^{[6]}$ and CAS (Coordinated Wakeup Scheduling) ${ }^{[7]}$ have been proposed to mitigate adverse effects of asynchronous duty cycles on end-to-end transmission reliability and latency. These protocols and mechanisms address the

Regular Paper

This work is supported in part by the International Science and Technology (S\&T) Cooperation Program of China (ISTCP) under Grant No. 2013DFA10690, and the National Natural Science Foundation of China (NSFC) under Grant Nos. 61672498 , 61303246 and 61100180 .

(C)2017 Springer Science + Business Media, LLC \& Science Press, China 
challenges of lossy links and asynchronous duty cycles in two layers, particularly in routing layer and MAC layer respectively. Although it is easier to conquer lossy links and asynchronous duty cycles in a layered approach, it induces separate control overheads in different layers, i.e., the overhead of estimating the link quality for the routing protocols and the overhead of synchronizing duty cycles for the MAC protocols. The cumulative overheads of these protocols make the current protocol stack for low-duty-cycle WSNs hard to scale with the network size ${ }^{[8]}$.

In this paper, we aim to address the challenges with a light-weight routing protocol, based on the low-powerlistening MAC protocol for low-duty-cycle WSNs. Opportunistic forwarding is a kind of stateless routing protocols, which make nodes forward packets without proactively established routing information. Instead, it determines the relay node at each hop on-the-fly by the sender based on some local network information or by conducting a contention process at the receiver side. Thus opportunistic forwarding behaves naturally in accordance with temporally changing interconnection relationships, which mostly stem from dynamic link qualities and asynchronous duty cycles, among nodes in WSNs. Therefore some previous studies, such as DSF (Dynamic Switch Forwarding) ${ }^{[9]}$ and C-MAC (Convergent MAC) ${ }^{[10]}$, have been proposed to exploit opportunistic forwarding to ensure reliable and timely data transmission in low-duty-cycle WSNs in an energy efficient manner.

Briefly speaking, DSF calculates delivery latency incurred from both asynchronous duty cycles and lossy links at each hop, and takes it as one of metrics to determine the best forwarder. C-MAC adopts normalized latency, which is the latency of each relay normalized by its geographical routing progress, as the metric to select the forwarder. Choice of forwarder set and forwarder priority can have significant impacts on the performance of opportunistic forwarding, in terms of packet delivery ratio (PDR) and packet delivery latency (PDL). Chau and Basu ${ }^{[11]}$ analyzed the latency of opportunistic forwarding in duty cycled WSNs as random walk on finite graphs. In [12], the authors proposed a method to minimize delay through joint control of wake-up pattern and forwarding priority. SF (simplified forward $)^{[13]}$ selects the node which wakes up and makes a progress of more than a threshold as the forwarder, so as to optimize forwarding delay. In [14], the authors proposed a series of preamble length control guidelines to minimize energy and latency cost.
These currently proposed opportunistic forwarding protocols and related optimization mechanisms lay a good foundation to achieve reliable data transmission with restrictions of delivery latency and energy consumption in low-duty-cycle WSNs. However, these protocols involve complicated mechanisms to determine the best forwarder, which usually incur heavy overheads for the resource-constrained nodes to maintain local network information or to conduct a contention in WSNs. Hence, we propose a light-weight opportunistic forwarding protocol, named LWOF, which does not need additional information or contention procedure to determine the best forwarder during data transmission. Specifically, LWOF exploits the analytical result about best forwarding area in [15] and takes advantage of the preamble sent by a low-power listening (LPL) MAC protocol (e.g., B-MAC ${ }^{[16]}$ ), which is widely used in wireless sensor networks with asynchronous duty cycles, to timely forward sensed data to a unique downstream node towards the sink. At the same time, we tune the preamble length of LPL MAC protocol to achieve energy efficiency and a specific forwarding probability, according to node density and sleep duration. The main contributions of the paper are as follows.

1) A light-weight opportunistic forwarding scheme (LWOF) is proposed to provide reliable and timely data delivery for wireless sensor networks with asynchronous duty cycles. It confines forwarding candidates to an optimized area, and hence can easily forward a packet to a unique downstream node towards the sink with a high probability, without the need of making a forwarding decision at each hop.

2) The preamble length of LPL MAC protocol is optimized by exploiting the non-deterministic characteristic of opportunistic forwarding, according to forwarding probability, node density and sleep duration. To the best of our knowledge, this is the first work that makes a theoretical analysis on the relationship among delivery probability, preamble length, node density and sleep duration for opportunistic forwarding in low-duty-cycle WSNs. The LPL MAC protocol with reduced preamble length is named as light-weight MAC protocol (LWMAC).

3) Performance of the proposed protocols is jointly evaluated through extensive simulations, in terms of packet delivery ratio, delivery latency, and normalized energy consumption (Joules per packet). Simulation results show that our proposed protocols can ensure a high successful ratio of packet delivery as expected and low delivery latency as a receiver-based opportunistic 
forwarding protocol, while reducing energy consumption per packet by at least twice.

The rest of the paper is organized as follows. Section 2 gives an overview of related work. Section 3 describes the design of opportunistic forwarding. Section 4 optimizes the preamble length of LPL MAC protocol through deriving relationship among delivery probability, preamble length, node density and node sleep duration. Section 5 evaluates our proposed schemes via simulations, and Section 6 makes a conclusion.

\section{Related Work}

Opportunistic forwarding protocols were initially proposed for mobile wireless ad-hoc networks with dynamic topology to improve packet delivery ratio (PDR) and reduce packet delivery latency (PDL). These protocols can be categorized into following two types according to their different ways to determine the best forwarder. One type uses the local network information of making a forwarding decision at the transmitter side, which is referred to as sender-based opportunistic forwarding. The other type uses a contention process to select a forwarder at the receiver side, which is referred to as receiver-based opportunistic forwarding.

Sender-Based Opportunistic Forwarding. Based on the historical network information (e.g., geographical positions, duty cycles, and connectivity probabilities of the neighbor nodes) or instantly probed information, the sender selects one node as the forwarder of the packet prior to data transmission. GPSR ${ }^{[17]}, \mathrm{EEF}^{[18]}$, $\mathrm{EGR}^{[19]}$, and $\mathrm{SDF}^{[20]}$ are examples of such kind of forwarding protocols. In [21], the authors proposed Sidewinder, which is a predictive data forwarding protocol, to handle intensive topology changes in mobile wireless sensor networks. It makes forwarding decision based on the distributed knowledge of a mobile sink location, which is updated by the Sequential Monte Carlo (SMC) prediction approach. In [22], the authors designed a Markov decision process based geographic routing protocol to achieve a desired successful delivery ratio and a bounded end-to-end delay.

Receiver-Based Opportunistic Forwarding. In such schemes, it is the receiver, rather than the sender, who is responsible for determining the forwarder of the packet. Most of the currently proposed protocols are of this kind, including $\mathrm{GeRaF}^{[23]}, \mathrm{CBF}^{[24]}, \mathrm{IGF}^{[25]}$, $\mathrm{SGF}^{[26]}$, and $\mathrm{ROF}^{[27]}$. They employ a timer-based contention process to make the forwarding decision. In particular, when a node has a packet to be forwarded to the next hop, it will broadcast a message (e.g., Request To Send (RTS)) to announce the forwarding demand. Each active neighbor node will determine the backoff time to reply to the demand, based on its own local information such as geographical position and available energy. The one with the shortest backoff will be chosen as the forwarding node. For $\mathrm{ExOR}^{[28]}$, the receiver makes the forwarding decision based on the prior knowledge of the network topology.

Recently, opportunistic forwarding has also been proposed for low-duty-cycle WSNs to improve RDR and reduce PDL. All these forwarding protocols are receiver-based. $\mathrm{DSF}^{[9]}$ is the first protocol which applies opportunistic forwarding mechanism in low-dutycycle WSNs with lossy links to achieve network energy efficiency, reliability, and timeliness in an integrated fashion. More recently, an opportunistic forwarding protocol, named $L^{2[29]}$, was proposed to further improve packet delivery ratio and reduce energy consumption of DSF through adapting link quality estimation to burstiness using multivariate Bernoulli link model. ORiNoCO ${ }^{[30]}, \mathrm{ORW}^{[31]}$ and ORPL ${ }^{[32]}$ all combine the collection tree protocol ${ }^{[5]}$ with opportunistic forwarding to increase packet delivery ratio and reduce delivery latency. In [33], the authors integrated opportunistic forwarding protocol with $\mathrm{X}-\mathrm{MAC}^{[34]}$ to further shorten strobed preambles of X-MAC.

The general idea of LWOF is essentially different from all the above protocols, in that it uses neither historical network information nor a contention process to select a forwarder prior to data transmissions. Through exploiting the analysis result about best forwarding area in [15], and confining forwarding candidates to an optimized area, LWOF makes any node in the forwarding area who first hears the preamble forward the packet. It removes the overhead of making a forwarding decision prior to data transmissions, while still ensuring reliable and timely packet delivery. Besides that, LWMAC and the LPL MAC protocols integrated with the currently proposed opportunistic forwarding protocols for low-duty-cycle WSNs differ in the way to reduce the energy for transmitting packets. In particular, LWMAC shortens the preamble of each packet in advance based on our deduced relationship among delivery probability, node density and sleep duration, while the currently proposed opportunistic forwarding protocols for low-duty-cycle WSNs all employ LPL MAC with strobed preambles, which are stopped transmitting to save energy immediately when the best forwarder receives the packet ${ }^{[35]}$. 


\section{LWOF Design}

In this section, we first present the network model and assumption for protocol design, and then elaborate the design of light-weight opportunistic forwarding for low-duty-cycle WSNs.

\subsection{Network Model and Assumption}

Sensor nodes are uniformly deployed in an area of $L \times W$ square meters, where $L$ and $W$ mean the length and the width of the area respectively. In the area, there is one sink to collect data from the sensor nodes. To save power, nodes alternate between active and sleeping states independently. In other words, each node works on its own duty cycle. This low-power operation of nodes induces the asynchronous communication problem in wireless sensor networks. An LPL MAC protocol, such as B-MAC ${ }^{[16]}$, that prefixes a preamble to each packet before sending it, is usually adopted to address the problem. As shown in Fig.1, prior to data transmission, a sender transmits a preamble lasting at least as long as the sleep period of the receiver, and other nodes wake up periodically to detect the arrival of packets. When a node wakes up and detects the preamble, it stays awake to receive data in the packet. In such a way, it guarantees normal communication among nodes, at the cost of bandwidth and energy spent in transmitting the preamble.

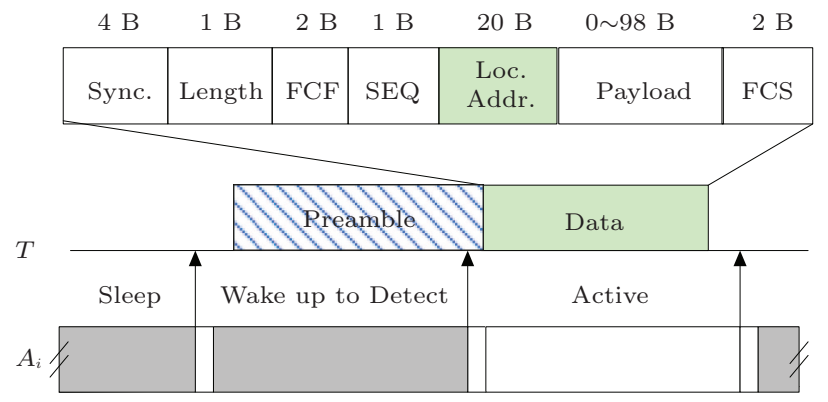

Fig.1. Packet transmission with an LPL MAC protocol (BMAC), where a sender $T$ transmits a packet led by a preamble and other nodes $A_{i}$ wake up periodically to detect the arrival of packets.

Each node obtains its location, denoted as ( $x$, $y$ ), through Global Positioning System (GPS) or selfconfiguring localization mechanisms ${ }^{[36]}$. The location of the sink is broadcasted to all sensor nodes during the network initialization phase. Packets are forwarded to the sink using the location-address semantics ${ }^{[17]}$, in which locations, instead of node IDs, are specified as the destination address. This location-address semantics is valid in many sensor networks, because sensor data, such as temperature readings, are normally tagged with the location information. Based on the format of data packet defined by IEEE 802.15.4, we can simply implement the location-address semantics by adapting the address field in the MAC layer header, as shown in Fig.1.

Each node is equipped with two radio interfaces and supports dual channel communication ${ }^{[37]}$. One is a low data rate channel for transmitting busy tone message, while the other is a higher data rate channel for transmitting sensor data. The former is referred to as the signal channel $\left(C_{\mathrm{s}}\right)$, while the latter is referred to as the data channel $\left(C_{\mathrm{d}}\right)$ in the following contents. The communication ranges of nodes in $C_{\mathrm{s}}$ and $C_{\mathrm{d}}$ are the same.

It is worth noting that the communication range of a node is assumed to be identical in all directions, as shown in Fig.2. Because links among nodes in WSNs are featured by non-isotropic radio connectivity in practice ${ }^{[38]}$, it would be more precise to model the radio transmission of nodes by taking into account the signal propagation characteristics, such as flat Rayleigh fading ${ }^{[39]}$. However, for the feasibility of making a theoretical analysis on the relationship among delivery probability, preamble length, node density and sleep duration for opportunistic forwarding in low-duty-cycle WSNs, we define the communication ranges of nodes in $C_{\mathrm{s}}$ and $C_{\mathrm{d}}$ to the distance between nodes which can get approximately $100 \%$ successful packet reception ratio, so as to leave out the outer irregular area of practical transmission model and make it approximate to our adopted disc model.

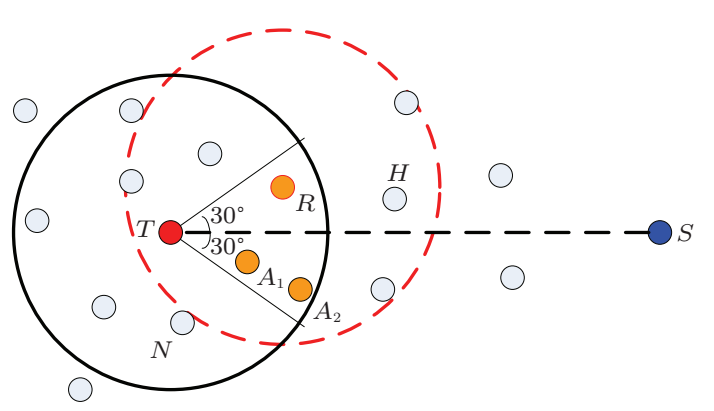

Fig.2. Illustration of a scenario where node $T$ is transmitting a packet towards sink $S$. $N$ represents the nodes located in the transmission range of $T, A$ represents the nodes located in the forwarding area, $R$ is the selected forwarder, and $H$ represents the hidden terminals for the transmission between $T$ and $R$. Communication range of $T$ in the data channel is denoted by solid line circle, and communication range of $R$ in the signal channel is denoted by dashed line circle. 


\subsection{Forwarding Scheme Design}

The basic design principle of opportunistic forwarding for low-duty-cycle WSNs is to select the unique best forwarder from an area of candidate nodes on the fly to deliver packets towards the sink node. Choice of the area of forwarding candidate nodes (i.e., forwarding area) can have impact on packet progress and complexity of forwarder selection. In particular, the larger the forwarding area, the higher the probability it can deliver packets with more progress. However, larger forwarding area incurs larger cost to make forwarding decision, particularly longer time to wait all the nodes in the forwarding area to wake up. Therefore, it is challenging to determine the forwarding area in a prior. According to the work done by Chen et al. ${ }^{[15]}$, a 60 degree radian area can achieve the same network performance as the maximum forwarding area when the average number of neighbors is larger than a threshold. Considering that sensor networks are usually densely deployed $^{[40]}$, we confine forwarding candidate nodes to a 60 -degree radian area. Fig. 2 gives an illustration of the forwarding area, which is within a 30 -degree radian area around the line connecting the sender and the sink on both sides.

With the forwarding area determined, the policy of selecting the best forwarder from the candidate nodes is another crucial factor in designing opportunistic forwarding scheme. As shown in Fig.2, for node $T$, which is transmitting packets towards sink $S$, at the current hop it has three forwarding candidates $A_{1}, A_{2}$, and $R$, which are denoted as black circles located within the radian area. The objective of forwarder selection is to make each packet delivered with the highest progress and reliability towards the sink, so as to ensure low delivery latency and high delivery ratio at the end. Considering that in low-duty-cycle WSNs the candidate node with the highest progress is not always on duty, which will incur additional latency (i.e., sleep latency) and transmission overhead (i.e., longer preamble before data packet), we take distance progress (Distance_progress $A_{A_{i}}$ ) and sleep latency (Sleep_latency $A_{i}$ ) into accounts when designing the policy of forwarder selection. The metric used in selecting the best forwarder is formulated as follows:

$$
\text { Velocity }_{A_{i}}=\frac{\text { Distance_progress }_{A_{i}}}{\text { Sleep_latency }_{A_{i}}} .
$$

With this metric, we can allow the transmitter to select the node, or allow the receiver to conduct a con- tention process to elect the node with the highest velocity as the forwarder. However, as pointed out in the introduction section of this paper, these methods require each node to collect and maintain information about neighbors, particularly duty cycles of neighbors, which will incur high overheads for resource-constrained WSNs. We see that distance progresses made by the nodes in the forwarding area do not differ so much. Therefore, it is very highly probable that the node waking up earliest can be the best candidate. Furthermore, we observe that in low-duty-cycle WSNs, all the candidate nodes wake up asynchronously, and the preamble sent by the transmitter cannot be detected by all the neighbor nodes at the same time. This observation is confirmed by the following theorem.

Theorem 1. In a wireless sensor network with asynchronous duty cycles, the probability that more than one node wakes up simultaneously is almost zero.

This theorem is based on the assumption that nodes wake up independently and the phase difference between two asynchronously duty-cycled nodes is exponentially distributed. We will give a strict proof of the above theorem in Subsection 4.2. Based on the above analysis, we design a light-weight opportunistic forwarding scheme, named LWOF, which makes a forwarding decision without local network information or a contention process. In particular, it takes advantage of the confined forwarding area and sequential detection of the preamble to reliably forward a packet to a unique downstream node on the fly.

We introduce the detail of LWOF design with an example illustrated in Fig.2. When node $T$ initiates a packet transmission, the following steps are taken to forward the packet to a unique downstream node towards the sink.

1) Packet Transmission. When node $T$ senses the signal channel idle, it starts transmitting the packet with a preamble in the data channel. The preamble contains the sender's and the sink's locations, and is interleaved with the synchronization bits (e.g., 0xAA or 0x55) in the traditional LPL MAC protocol.

2) Preamble Detection and Packet Reception. In the communication range of the sender $T$, and any node $N$ who wakes up and detects the preamble of the packet, does the following computation to determine whether it is located in the 60-degree radian area, using the sender's location and the sink's location included in the preamble, and its own location.

$$
\text { Degree }_{\angle N T S}=\operatorname{acos}\left(\frac{|T N|^{2}+|T S|^{2}-|N S|^{2}}{2|T N||T S|}\right),
$$


where $|T N|,|T S|$ and $|N S|$ represent the Euclidean distances among the sender $T$, the sink $S$, and the node $N$. If the angle $\angle N T S$ is larger than 30 degrees, the node will turn off its data radio. In such a way, the gray nodes $N$ within the communication range of $T$, but outside of the 60-degree radian area, are deprived of forwarding rights. For the node who wakes up and judges that $\angle N T S$ is no larger than 30 degrees, it will send a busy tone in the signal channel immediately, and occupy the signal channel until the packet is received. Others sensing the busy tone will keep sleeping until the next scheduled waking up. As shown in Fig.2, node $R$ in the 60-degree radian area wakes up and detects the preamble. Thus it sends a busy tone in the signal channel, and nodes $A_{i}$ sensing the busy tone will keep on sleeping. The hidden terminal $H$ will defer sending until it senses the signal channel idle.

It is worth pointing out that the function of the busy tone in LWOF is two-fold. Firstly, it is to solve the hidden terminal problem as described above. Node $H$ sensing the busy tone in $C_{\mathrm{s}}$ will defer data transmission to prevent themselves from breaking down the transmission between $T$ and $R$ in $C_{\mathrm{d}}$. Secondly, it is to prevent duplicate forwarding. Within the 60 -degree radian area, the distance between any two nodes is smaller than the communication range of $R$, and thus the busy tone sent by the forwarder $R$ can be detected by any other nodes waking up later and serves as a signal indicating that the packet will be forwarded by $R$. According to Theorem 1 , the probability that more than one node sends busy tone in $C_{\mathrm{s}}$ simultaneously is very low, thereby it makes sense to assume that no collision will occur when $R$ transmits busy tone in $C_{\mathrm{s}}$.

3) No Acknowledgement. We consider that sensor nodes are uniformly deployed with high density ${ }^{[40]}$, and thus the void problem (i.e., the absence of nodes in the forwarding area $)^{[17]}$ hardly occurs. In case of packet transmission failure due to path loss, we can let $R$ send a negative acknowledgment (NACK) to $T$ in the signal channel, if $R$ does not receive the packet successfully. When $T$ receives a NACK, it will retransmit the packet. However, we assume that node $R$ detecting the preamble has a high probability of receiving the packet successfully, in that the pair of nodes $(T, R)$ exclusively reserves the data channel during packet transmission through sending a busy tone simultaneously in the signal channel. Thus we eliminate the acknowledgement to reduce the cost of packet transmission at each hop.

In summary, each node takes actions as shown in Algorithm 1, when it wakes up to forward packets timely and reliably. From the pseudocode of LWOF, we can see that the time complexity and the space complexity of the algorithm are both $O(1)$. Thus it is light-weight and feasible to run on sensor nodes with limited computing and memory resources.

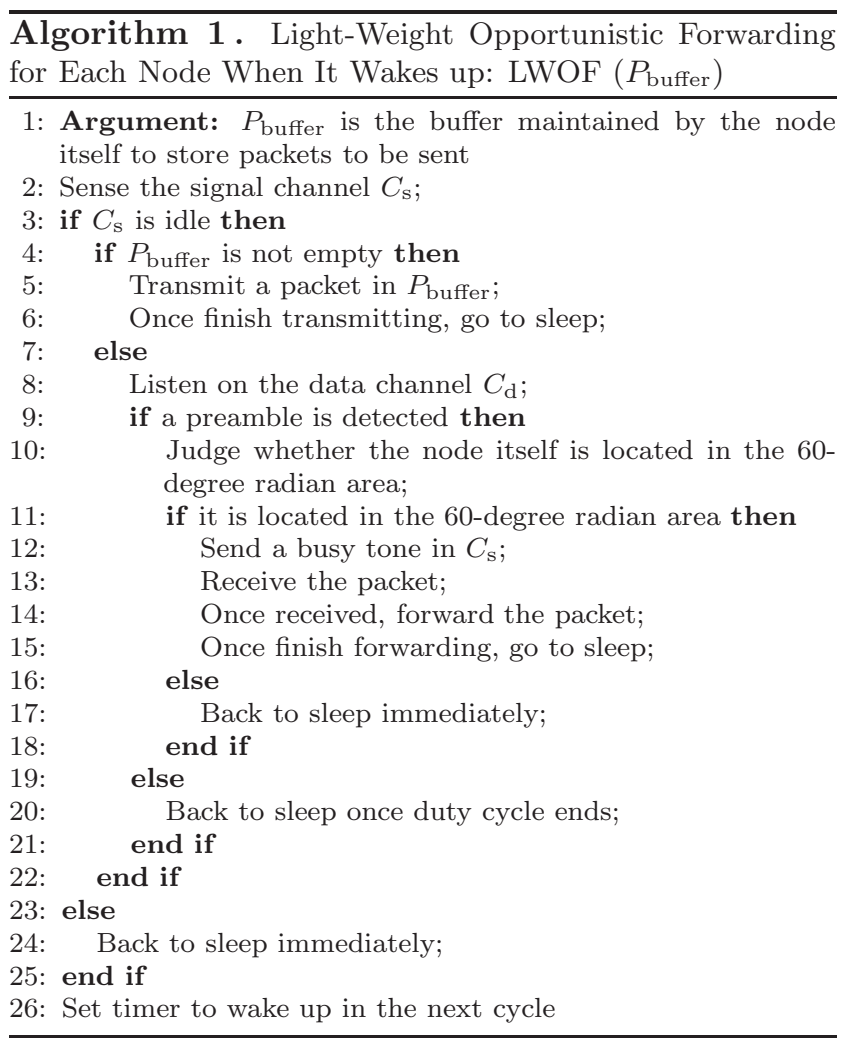

\section{LPL MAC Optimization Under LWOF}

\subsection{Overview of LPL MAC Under LWOF (LWMAC)}

We note that the LPL MAC protocol is originally designed for the unicast in wireless sensor networks with asynchronous duty cycles. In other words, packets are forwarded to a specific node at each hop, but the transmitter does not know when the next-hop node will wake up. Thus it must employ a sufficiently long preamble to ensure that the packet is detected by the next hop. When the data rate is fixed, the preamble length increases linearly with the sleep duration, i.e., the original preamble length measured in bits is $\left(T_{\mathrm{s}} \times R_{\mathrm{d}}\right)$, where $T_{\mathrm{s}}$ is the sleep duration and $R_{\mathrm{d}}$ is the data rate.

We exploit the non-deterministic characteristic of opportunistic forwarding to optimize the preamble length of the LPL MAC protocol. The optimized LPL MAC protocol, named LWMAC, employs a much shorter preamble than the original one. Taking the sce- 
nario shown in Fig. 2 for example, node $T$ transmits packets with a preamble of length shorter than $\left(T_{\mathrm{S}} \times R_{\mathrm{d}}\right)$. Because nodes in low-duty-cycle WSNs wake up asynchronously, node $A_{1}$ in the forwarding area may not detect the preamble when it wakes up before $T$ starts transmitting packets, but another node $A_{2}$ waking up $\tau$ time units later than $A_{1}$ may detect it. For LWOF, since any node that first detects the preamble can be the forwarder of the packet, the packet with a shorter preamble can still be forwarded. The above described process of packet forwarding with LWOF and LWMAC can be predicted in Fig.3.

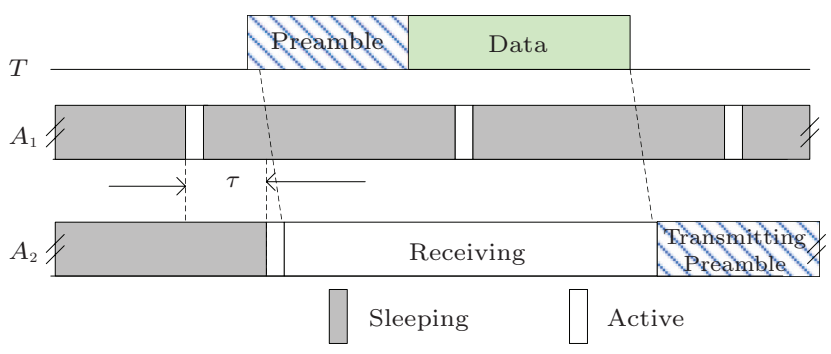

Fig.3. Packet transmission with LWMAC protocol, which employs a shorter preamble than the LPL protocol (B-MAC). The preamble is not detected by $A_{1}$, but it is detected by $A_{2} \tau$ time units later. $A_{2}$ receives and forwards the packet.

It is worth noting that later detection of the shortened preamble will not increase the delivery latency of a packet, because the transmission of data part of a packet cannot be started until the transmission of preamble ends. Thus whenever the preamble is detected, it will not incur additional delay for packet forwarding, instead it will reduce energy consumed in sending the preamble. However, if a preamble is too short, no node in the forwarding area can detect it, which leads to a void problem. Therefore the question that should be addressed in Subsection 4.2 is how long a preamble is sufficient for making LWOF forward packets successfully with a high probability.

\subsection{Optimizing Preamble Length for LWMAC}

Now we analyze the relationships among forwarding probability, node density, preamble length, and sleep duration. As shown in Fig.2, $N_{f}$ nodes in the forwarding area wake up asynchronously after sleeping for $T_{\mathrm{s}}$ time units. We assume that the phase difference between two asynchronously duty-cycled nodes is an exponentially distributed random variable with average $T_{\mathrm{s}} / N_{f}$. Thus we can view the sequence of nodes' waking up as a Poisson process, and the probability that more than one node wakes up in a period $t$ can be formulated as follows.

$$
\begin{aligned}
P_{t}(i>1) & =1-P_{t}(i=0)-P_{t}(i=1) \\
& =1-e^{-\frac{N_{f}}{T_{\mathrm{s}}} t}-\frac{N_{f}}{T_{\mathrm{s}}} \times t \times e^{-\frac{N_{f}}{T_{\mathrm{s}}} t} \\
& =1-\left(1+\frac{N_{f}}{T_{\mathrm{s}}} \times t\right) \times e^{-\frac{N_{f}}{T_{\mathrm{s}}} t} .
\end{aligned}
$$

From the above equation, we can get that

$$
P_{t \rightarrow 0}(i>1) \rightarrow 0 \text {. }
$$

In particular, when the period $t$ is extremely short (approaching zero), $P_{t}$ approaches zero. In other words, the probability the more than one node wakes up simultaneously is almost zero. This proves Theorem 1 given in Subsection 3.2.

For the same reason, we can get the forwarding probability $P_{f}$ that at least one node wakes up in a period $T_{p}$, where $T_{p}$ is the length of the preamble.

$$
\begin{aligned}
P_{f} & =P_{t=T_{p}}(i \geqslant 1)=1-P_{t=T_{p}}(i=0) \\
& =1-e^{-\frac{N_{f}}{T_{\mathrm{s}}} T_{p}} .
\end{aligned}
$$

For LWOF, the forwarding area is a 60-degree radian area. Besides, according to the network model defined in Subsection 3.1, nodes are uniformly deployed. Thus the number of nodes in the forwarding area, i.e., $N_{f}$ in (1), can be formulated as follows.

$$
N_{f}=\frac{\pi r^{2}}{6} D
$$

where $D$ is the node density, and $r$ is the communication range of nodes in the data channel.

From (1) and (2), we can formulate the length of the preamble as a function of forwarding probability, node density and sleep duration, as shown in (3).

$$
T_{p}=\min \left(-\ln \left(1-P_{f}\right) \frac{6 T_{\mathrm{s}}}{\pi r^{2} D}, T_{s}\right) .
$$

Referring to (3), we can tune the preamble length of LWMAC protocol for a deployed sensor network with the specified node density and sleep duration, in order to achieve a specific forwarding probability. As shown in Fig.4, for a sensor network with communication range of 20 meters, node density of 0.03 , and sleep duration of $135 \mathrm{~ms}$, its preamble length can be set accordingly to achieve expected forwarding probability. We can see that as the expected forwarding probability decreases, the preamble length can be reduced accordingly. Furthermore, to achieve an expected forwarding 
probability, the preamble length can be reduced with increasing node density. In addition, LWMAC always employs a shorter preamble than the current LPL MAC protocol (B-MAC).

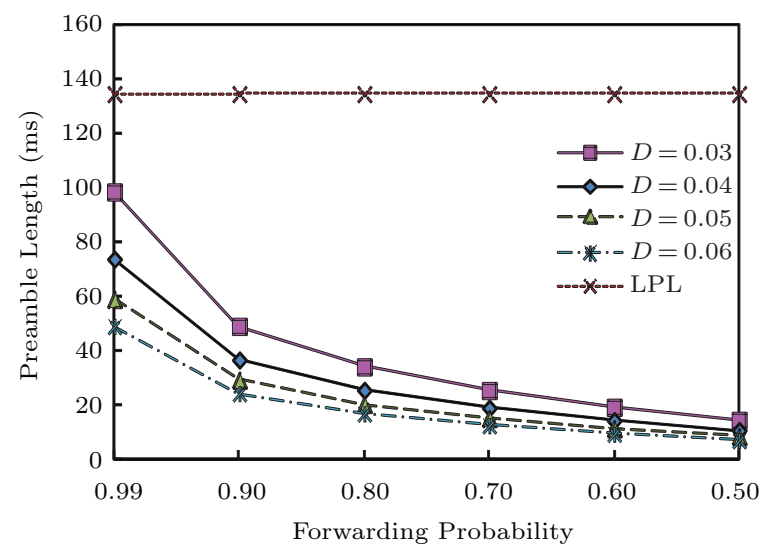

Fig.4. Relationship between the preamble length and the expected forwarding probability at each hop for LWMAC with different node densities, and fixing sleep duration to $135 \mathrm{~ms}$.

Fig.5 illustrates the relationship between the preamble length and the node density for LWMAC with different forwarding probabilities. Here we specify the sleep duration $T_{\mathrm{s}}$ to $135 \mathrm{~ms}$ and the radio range $r$ to 20 meters. We can see that LWMAC takes advantage of the increase in node density to shorten its preamble. In other words, as more nodes are uniformly deployed in the field, the preamble length can be reduced while guaranteeing the same forwarding probability.

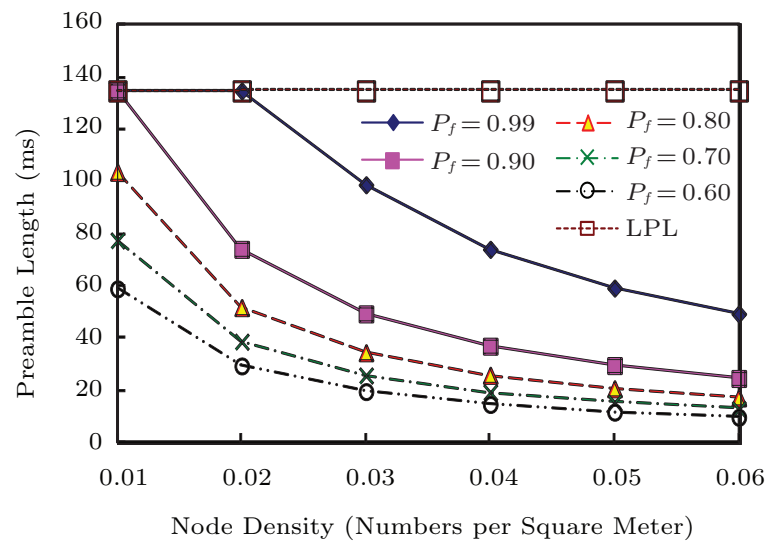

Fig.5. Relationship between the preamble length and the node density for LWMAC with different forwarding probabilities, and fixing sleep duration to $135 \mathrm{~ms}$.

Fig.6 illustrates the relationship between the preamble length and the sleep duration for LWMAC with different forwarding probabilities, when the node density is fixed to 0.03 and the communication range $r$ is 20 meters. We can see that like the LPL MAC protocol, the preamble length of LWMAC is linearly proportional to the sleep duration, but it is much smaller than that of the LPL MAC protocol.

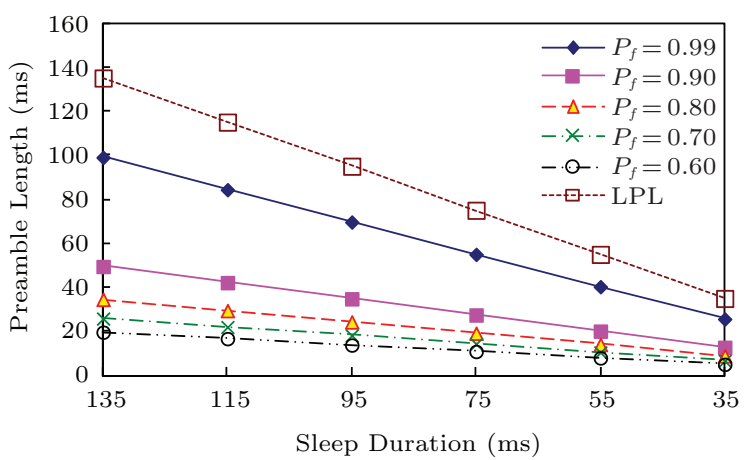

Fig.6. Relationship between the preamble length and the sleep duration for LWMAC with different forwarding probabilities, and fixing node density to 0.03 .

\section{Performance Evaluation}

To evaluate the performance, we implement the light-weight opportunistic forwarding (LWOF) protocol, along with the energy-efficient MAC (LWMAC) protocol in a commonly-used wireless network simulator $(\text { GloMoSim })^{[41]}$. For comparison, the LPL MAC (B-MAC) protocol is implemented as a special case of the LWMAC protocol, which employs a preamble of fixed length equal to the sleep duration. In addition, the ROF protocol ${ }^{[27]}$ is implemented for the purpose of comparison, because it also uses a dualchannel communication mechanism to avoid data collision and duplicated forwarding. However, as described in Section 2, ROF is a receiver-based opportunistic forwarding scheme, which employs a forwarding right contention process to determine the best forwarder. It should be pointed out that ROF was originally designed without considering the low duty cycle in sensor networks. We adapt ROF to work in low-duty-cycle WSNs, by making each node maintain its neighbors' information about duty cycling. The simulation scenario is described in detail below.

\subsection{Simulation Setups and Metrics}

Settings of some important simulation parameters are listed in Table 1. We put 300 nodes uniformly in a square region of 100 meters by 100 meters. The communication range of each node is 20 meters, and thus the expected distance from the source to the sink is about 7 hops. The maximum data rate of the data channel is $38.4 \mathrm{Kbps}$. Each node turns its own data radio on and off independently. In particular, nodes keep active 
and sense the data channel for $8 \mathrm{~ms}$ after sleeping for a period of time (e.g., $135 \mathrm{~ms}, 115 \mathrm{~ms}, 95 \mathrm{~ms}, 75 \mathrm{~ms}$, $55 \mathrm{~ms}$ and $35 \mathrm{~ms})$. For LWOF, the expected forwarding probability $\left(P_{f}\right)$ is 0.9 , and the preamble length of LWMAC is set according to (3) with the node density fixed to 0.03 . The energy consumption model is established based on the measurement results presented in [42]. In particular, the current for transmission is 8.5 $\mathrm{mA}$, while it is $7.0 \mathrm{~mA}$ for data reception and listening to data channel. For LWOF, the signal radio consumes about $100 \mu \mathrm{A}$ current when the node turns off the data radio.

Table 1. Settings of Simulation Parameters

\begin{tabular}{ll}
\hline Simulation Parameter & Setting Value \\
\hline Region's area $\left(\mathrm{m}^{2}\right)$ & $100 \times 100$ \\
Number of nodes & 300 \\
Radio range of nodes in both $C_{\mathrm{d}}$ and $C_{\mathrm{s}}$ & 20 \\
$(\mathrm{~m})$ & \\
Physical data rate of nodes in $C_{\mathrm{d}}$ & 38.4 \\
(Kbps) & \\
Active duration of nodes in $C_{\mathrm{d}}(\mathrm{ms})$ & 8 \\
Sleep duration of nodes in $C_{\mathrm{d}}(\mathrm{ms})$ & $135 \sim 35$ \\
Preamble length of LWMAC & Shown in Fig.6 with \\
& $P_{f}=0.9$ \\
Preamble length of LPL & Equal to sleep dura- \\
& tion \\
Electric current for data transmission in & 8.5 \\
$C_{\mathrm{d}}(\mathrm{mA})$ & \\
$\begin{array}{l}\text { Electric current for data reception in } C_{\mathrm{d}} \\
\text { (mA) }\end{array}$ & 7.0 \\
$\begin{array}{l}\text { Electric current for busy tone transmis- } \\
\text { sion and reception in } C_{\mathrm{s}}(\mu \mathrm{A})\end{array}$ & 100 \\
\hline
\end{tabular}

We evaluate the following three protocols, namely ROF, LWOF with the LPL MAC protocol (LWOFLPL), and LWOF with the LWMAC protocol (LWOFLWMAC) in terms of the following metrics:

1) Packet delivery ratio: the number of packets received by the sink divided by the number of packets transmitted by the source;

2) Delivery latency: the average delay required for forwarding a packet from the source to the sink;

3) Energy consumption per packet: the total energy consumed for all the nodes when the simulation ends divided by the number of packets received by the sink.

\subsection{Performance with Single Sensing Data Flow}

We specify the two nodes, which lie at the two ends of the diagonal of the region, as the data source and the sink respectively. The source generates a packet of
36 bytes every one minute, and runs for 24 hours. Because the nodes are randomly deployed, different random seeds can generate different topologies of node deployment. Results shown in this subsection are averages over five runs of the simulation with different random seeds. The error bars in Fig.7 Fig.10 show 95\% confidence intervals for these results.

First, we check whether LWOF can forward packets with the probability as expected. Therefore, we calculate the ratio of the total number of data packets sent (or forwarded) by all the nodes to the total number of data packets received by all the nodes in the data channel. Fig.7 shows the results, which can be seen as the averaged packet delivery ratio at each hop. We can see that all these opportunistic forwarding protocols can successfully deliver about $90 \%$ packets to the next hop, since they all employ a signal channel to ensure the successful reception of packets by the next hop. Besides, we can see that the LPL MAC protocol with optimized preamble length performs well under LWOF.

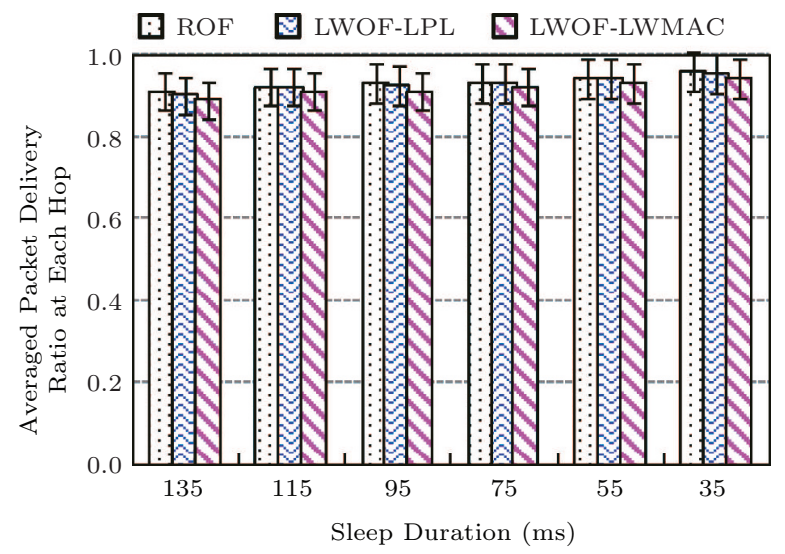

Fig.7. Averaged packet delivery ratio at each hop for different forwarding schemes in sensor networks with different duty cycles (one sensing flow).

Fig. 8 shows the packet delivery ratios of different forwarding schemes for the sensor networks with different duty cycles. We can see that ROF and LWOF-LPL achieve a higher delivery ratio than LWOF-LWMAC. For ROF, due to the inaccuracy of the historical information about the neighbors' duty cycles, some packets may be lost by the intermediate nodes. For LWOF-LPL and LWOF-LWMAC, failed delivery is mainly due to the lack of consideration of link quality when selecting the forwarder, and the lack of acknowledgment mechanism to recover the lost packets. However, the induced packet loss is not so severe for LWOF-LWMAC, because the node that can parse the location information in the preamble correctly has a high probability to receive the packet successfully. 


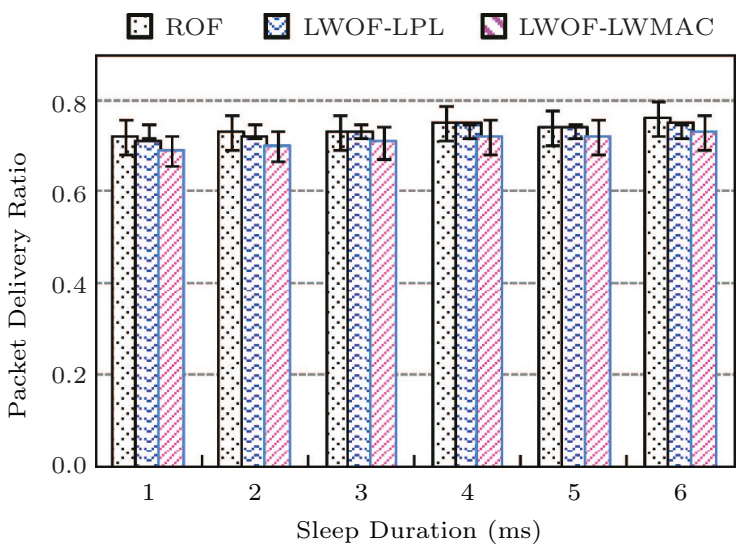

Fig.8. Packet delivery ratio of different forwarding schemes for sensor networks with different duty cycles (one sensing flow).

Fig.9 compares the packet delivery latency of different forwarding schemes. As introduced at the beginning of Section 5, the difference between LWOF-LPL and LWOF-LWMAC lies in their adopted MAC protocols. In particular, LWOF-LPL adopts the existing LPL MAC protocol which employs a preamble of fixed length equal to the sleep duration, while LWOFLWMAC adopts MAC protocol with reduced preamble length according to our deduced relationship shown in Fig.6. The long preamble employed by LWOF-LPL causes a higher transmission delay than that by LWOFLWMAC. Since ROF employs a contention process to select the forwarder node, in theory it can delay packets along a shorter path than LWOF-LWMAC. However, the delay incurred from the contention process trades off the benefits brought by the more optimized selection of the forwarder. Thus ROF outperforms LWOFLWMAC very marginally in terms of delivery latency.

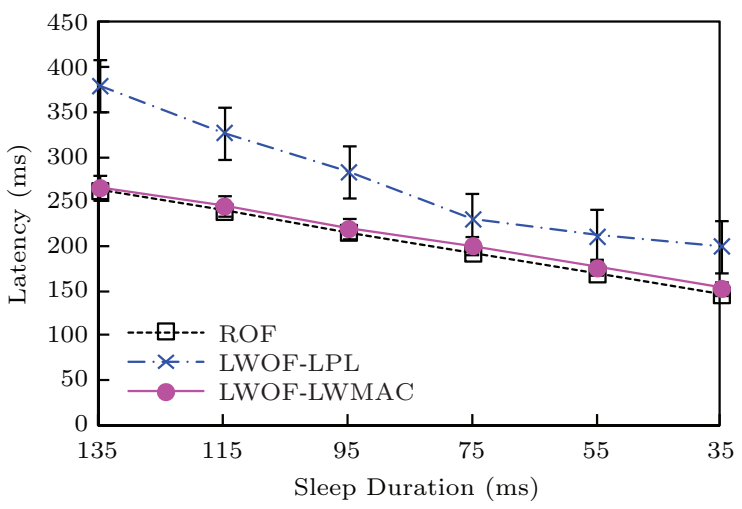

Fig.9. Delivery latency of different forwarding schemes for sensor networks with different duty cycles (one sensing flow).

As shown in Fig.10, as nodes wake up more frequently, energy consumed for delivering each packet increases, since more energy is consumed in listening to the data channel. However, for LWOF-LWMAC, its reduced preamble compensates for more energy consumed with a higher duty cycle. Thus average energy consumed for delivering each packet increases only a little with lower sleep duration for LWOF-LWMAC, whereas the longer preamble employed in LWOF-LPL results in much more energy consumption for transmitting each packet. For ROF, extra energy is consumed in periodic exchanges of duty cycling information and contention process, and it is less energy-efficient than LWOF-LWMAC.

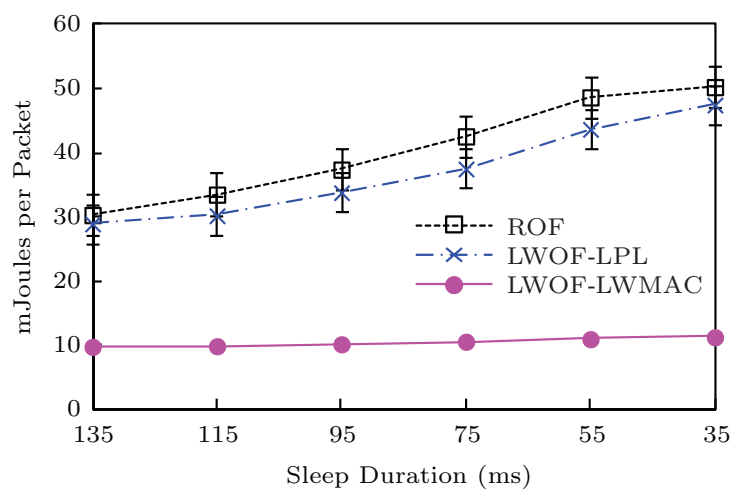

Fig.10. Normalized energy consumption of different forwarding schemes for sensor networks with different duty cycles (one sensing flow).

\subsection{Performance with Multiple Sensing Data Flows}

To evaluate the performance of our proposed opportunistic forwarding protocol in low-duty-cycle WSNs with dense data flows, we specify the three nodes at the three corners of the square area as the sensor nodes generating sensing data packets of 36 bytes every minute. Like the previous simulations, the sink node is located at the other corner of the square area, and every run of simulation lasts for 24 hours. For a WSN with a specified duty cycle, its performance is averaged over five runs of simulations with different random seeds. The error bars in Figs.11 13 show 95\% confidence intervals for the results.

Fig.11 compares the ratios of packets received by the sink in WSNs with different duty cycles through different forwarding schemes. We can see that all these forwarding schemes can ensure almost the same high delivery ratio as in previous scenarios with only one sensing flow. As explained before, this mainly results from dual-channel communication. In addition, we can see that LWOF-LPL does not outperform LWOF-LWMAC so much in terms of delivery ratio. Thus we can know 
that the reduced preamble of LWMAC has little impact on the reliability of communication in low-duty-cycle WSNs under LWOF.

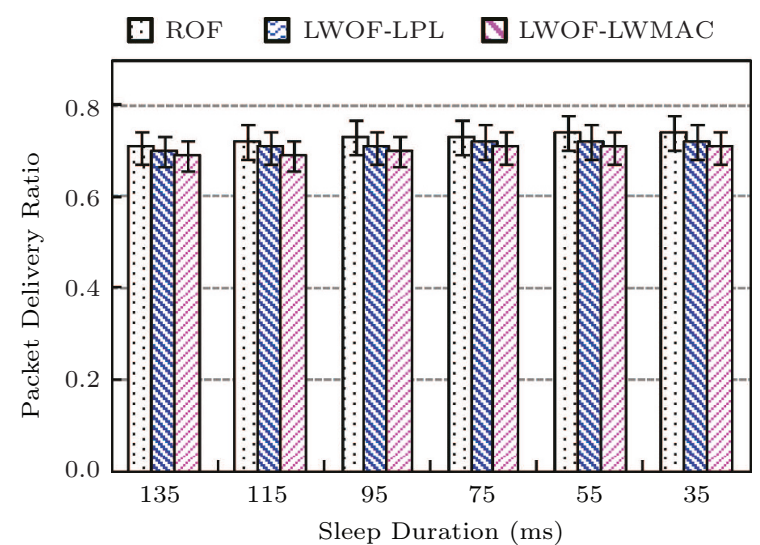

Fig.11. Packet delivery ratios of different forwarding schemes for sensor networks with different duty cycles (three sensing flows).

Fig.12 compares the delivery latency of ROF and LWOF-LWMAC, which shows that ROF has longer latency than LWOF-LWMAC. The longer latency of ROF is mainly due to the fact that it always selects the fixed set of nodes along the shortest path to the sink as the forwarder. As a result, in networks with ROF the channel access delay increases with hops close to the sink when more than one flow is generated from the same direction, and it trades off the benefit brought by forwarding packets along a shorter path than LWOF. In networks with LWOF-LWMAC, the forwarder is any node in the forwarding area who first hears the preamble, thereby traffics close to the sink are not so dense as that with ROF, and the channel access contention at hops close to the sink cannot be so severe as that with ROF. For LWOF-LPL, the longer preamble leads to longer transmission delay and channel access delay, and it forwards packets with the highest delay.

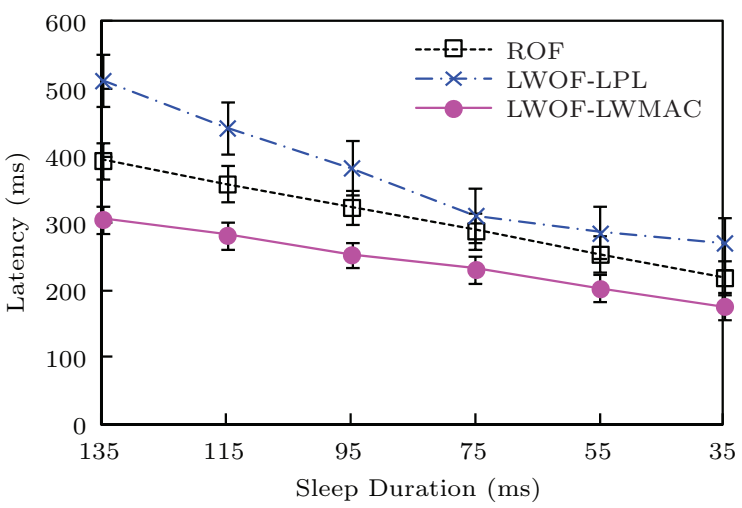

Fig.12. Delivery latency of different forwarding schemes for sensor networks with different duty cycles (three sensing flows).
Fig.13 compares energy consumed for each packet with different forwarding schemes. Because LWOFLWMAC adopts reduced preamble, which compensates for more energy consumed with decreased sleep duration, its energy consumption per packet increases only a little with a higher duty cycle. Besides, compared with Fig.10, we can see that the energy consumption for LWOF-LWMAC does not increase with the number of flows in networks. This is mainly due to the light weight design of our protocol, which has almost zero cost to determine the forwarder at each hop. For ROF, its energy consumption increases a little, which mainly results from more control packets exchanged among nodes to carry out the forwarder election process.

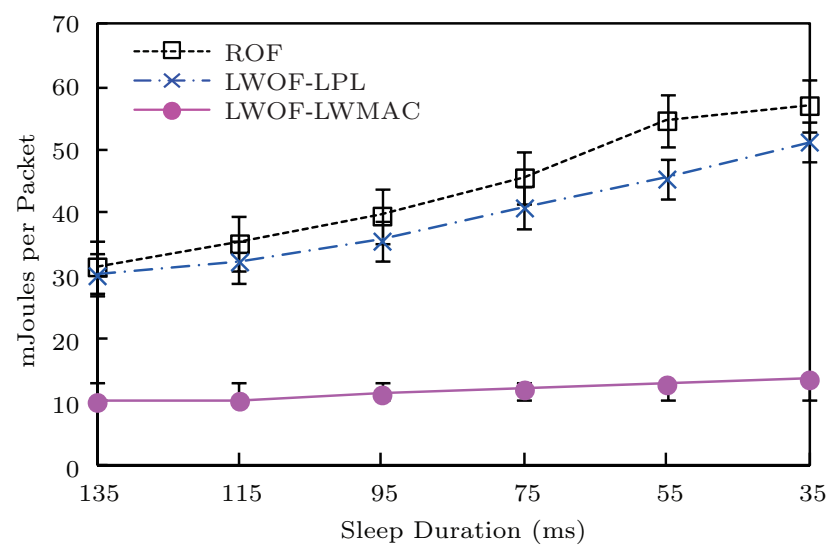

Fig.13. Normalized energy consumption of different forwarding schemes for sensor networks with different duty cycles (three sensing flows).

The above findings from our simulations show that our proposed light-weight opportunistic forwarding (LWOF) protocol, along with the low-power-listening MAC protocol with optimized preamble length (LW$\mathrm{MAC}$ ), can achieve relatively good performance in delivery reliability and latency, while it does not incur higher energy consumption in WSNs with different duty cycles.

\section{Conclusions}

In this paper, we proposed a light-weight opportunistic forwarding (LWOF) scheme to address the challenge of reliable and prompt data delivery in WSNs consisting of nodes with asynchronous duty cycles. Different from other recently proposed schemes, LWOF employs neither historical network information nor a contention process to select a forwarder prior to data transmissions. It confines forwarding candidates to an optimized area, and takes advantage of the preamble in LPL MAC protocols and dual-channel communication 
to remove the overhead of making a forwarding decision prior to data transmission.

Under the light-weight opportunistic forwarding, we made a theoretical analysis on the relationship among delivery probability at each hop, preamble length, node density and sleep duration. Based on the analysis result, we proposed an energy-efficient MAC protocol (LWMAC) with a shortened preamble, by exploiting the non-deterministic characteristics of opportunistic forwarding. The preamble length in LWMAC is a function of the delivery probability, node density and node sleep duration.

Simulation results showed that LWOF, along with LWMAC, could ensure packets delivered successfully with a ratio as expected. At the same time, it did not incur extra delay as compared with a receiver-based opportunistic forwarding scheme (ROF). Furthermore, LWOF can reduce the energy cost for delivering each packet as compared with ROF, while LWMAC reduces energy consumption per packet by at least twice as compared with traditional LPL MAC protocol.

\section{References}

[1] Dong W, Liu Y, He Y, Zhu T, Chen C. Measurement and analysis on the packet delivery performance in a large-scale sensor network. IEEE/ACM Transactions on Networking, 2014, 22(6): 1952-1963.

[2] Liang C J M, Priyantha B, Liu J, Terzis A. Surviving Wi-Fi interference in low power ZigBee networks. In Proc. the 8th ACM SenSys, Nov. 2010, pp.309-322.

[3] Kong L, Xia M, Liu X Y, Wu M Y, Liu X. Data loss and reconstruction in sensor networks. In Proc. IEEE INFOCOM, April 2013, pp.1654-1662.

[4] Hao J, Zhang B, Mouftah H. Routing protocols for duty cycled wireless sensor networks: A survey. IEEE Communications Magazine, 2012, 50(12): 116-123.

[5] Gnawali O, Fonseca R, Jamieson K, Moss D, Levis P. Collection tree protocol. In Proc. the 7th ACM SenSys, Nov. 2009, pp.1-14.

[6] Ye W, Silva F, Heidemann J. Ultra-low duty cycle MAC with scheduled channel polling. In Proc. the 4 th ACM SenSys, Oct. 31-Nov. 3, 2006, pp.321-334.

[7] Zhu Y, Liu Y, Ni L. Optimizing event detection in low dutycycled sensor networks. ACM Wireless Networks (WINET), 2012, 18(3): 241-255.

[8] Liu Y, He Y, Li M, Wang J, Liu K, Li X Y. Does wireless sensor network scale? A measurement study on GreenOrbs. IEEE Transactions on Parallel and Distributed Systems, 2013, 24(10): 1983-1993.

[9] Gu Y, He T. Data forwarding in extremely low duty-cycle sensor networks with unreliable communication links. In Proc. the 5th ACM SenSys, Nov. 2007, pp.321-334.
[10] Liu S, Fan K W, Sinha P. CMAC: An energy efficient MAC layer protocol using convergent packet forwarding for wireless sensor networks. In Proc. the 4th IEEE SECON Merged with IWWAN, Jun. 2007, pp.11-20.

[11] Chau C K, Basu P. Exact analysis of latency of stateless opportunistic forwarding. In Proc. the 28th IEEE INFOCOM, April 2009, pp.828-836.

[12] Kim J, Lin X, Shroff N. Optimal anycast technique for delay-sensitive energy-constrained asynchronous sensor networks. In Proc. the 28th IEEE INFOCOM, Apr. 2009, pp.612-620.

[13] Naveen K, Kumar A. Tunable locally-optimal geographical forwarding in wireless sensor networks with sleep-wake cycling nodes. In Proc. the 29th IEEE INFOCOM, March 2010, pp.920-928.

[14] Xue Y, Vuran M, Ramamurthy B. Cost efficiency of anycast-based forwarding in duty-cycled WSNs with lossy channel. In Proc. the 17th IEEE SECON, Jun. 2010 pp.520-528.

[15] Chen D, Deng J, Varshney P. On the forwarding area of contention-based geographic forwarding for ad hoc and sensor networks. In Proc. IEEE SECON, Sept. 2005, pp.130141.

[16] Polastre J, Hill J, Culler D. Versatile low power media access for wireless sensor networks. In Proc. the 2nd ACM SenSys, Nov. 2004, pp.95-107.

[17] Karp B, Kung H T. GPSR: Greedy perimeter stateless routing for wireless networks. In Proc. the 6th ACM MobiCom, Aug. 2000, pp.243-254.

[18] Seada K, Zuniga M, Helmy A, Krishnamachari B. Energyefficient forwarding strategies for geographic routing in lossy wireless sensor networks. In Proc. the 2nd ACM SenSys, Nov. 2004, pp.108-121.

[19] Lee S, Bhattacharjee B, Banerjee S. Efficient geographic routing in multihop wireless networks. In Proc. the 6th ACM MobiHoc, May 2005, pp.230-241.

[20] Larsson P, Johansson N. Multiuser diversity forwarding in multihop packet radio networks. In Proc. IEEE WCNC, Mar. 2005, pp.2188-2194.

[21] Keally M, Zhou G, Xing G. Sidewinder: A predictive data forwarding protocol for mobile wireless sensor networks. In Proc. the 6th IEEE SECON, June 2009.

[22] Hao J, Yao Z, Huang K, Zhang B, Li C. An energy-efficient routing protocol with controllable expected delay in dutycycled wireless sensor networks. In Proc. IEEE ICC, Jun. 2013, pp.6215-6219.

[23] Zorzi M, Rao R. Energy and latency performance of geographic random forwarding for ad hoc and sensor networks. In Proc. IEEE WCNC, Mar. 2003, pp.1930-1935.

[24] Füssler H, Widmer J, Käemann M, Mauve M, Hartenstein H. Contention-based forwarding for mobile ad hoc networks. Ad Hoc Networks, 2003, 1(4): 351-369.

[25] He T, Blum B, Cao Q, Stankovic J, Son S, Abdelzaher T. Robust and timely communication over highly dynamic sensor networks. Real-Time Systems, 2007, 37(3): 261-289.

[26] Huang P, Chen H, Xing G, Tan Y. SGF: A state-free gradient-based forwarding protocol for wireless sensor networks. ACM Transactions on Sensor Networks, 2009, 5(2): $14: 1-14: 25$ 
[27] Li L, Sun L, Ma J, Chen C. A receiver-based opportunistic forwarding protocol for mobile sensor networks. In Proc. the 28th IEEE ICDCS, June 2008, pp.198-203.

[28] Biswas S, Morris R. ExOR: Opportunistic multi-hop routing for wireless networks. ACM SIGCOMM Computer Communication Review, 2005, 35(4): 133-144.

[29] Cao Z, He Y, Liu Y. L²: Lazy forwarding in low duty cycle wireless sensor networks. In Proc. IEEE INFOCOM, Mar. 2012, pp.1323-1331.

[30] Unterschütz S, Renner C, Turau V. Opportunistic, receiverinitiated data-collection protocol. In Proc. the 9th EWSN, Feb. 2012, pp.1-16.

[31] Landsiedel O, Ghadimi E, Duguennoy S, Johansson M. Low power, low delay: Opportunistic routing meets duty cycling. In Proc. the 11th ACM IPSN, Apr. 2012, pp.185-196.

[32] Duquennoy S, Landsiedel O, Voigt T. Let the tree bloom: Scalable opportunistic routing with ORPL. In Proc. the 11th ACM SenSys, Nov. 2013, pp.2:1-2:14.

[33] Autenrieth M, Frey H. PaderMAC: A low-power, lowlatency MAC layer with opportunistic forwarding support for wireless sensor networks. In Proc. the 10th ADHOCNOW, July 2011, pp.117-130.

[34] Buettner M, Yee G, Anderson E, Han R. X-MAC: A short preamble MAC protocol for duty-cycled wireless sensor networks. In Proc. the 4th ACM SenSys, Oct.31-Nov.3, 2006, pp.307-320.

[35] Chen H, Cui L, Li V O. Joint design of opportunistic forwarding and energy-efficient MAC protocol in wireless sensor networks. In Proc. IEEE GLOBECOM, Nov.3-Dec.4, 2009.

[36] Liu Y, Yang Z, Wang X, Jian L. Location, localization, and localizability. Journal of Computer Science and Technology (JCST), 2010, 25(2): 274-297.

[37] Ansari J, Zhang X, Mähönen P. Multi-radio medium access control protocol for wireless sensor networks. International Journal of Sensor Networks, 2010, 8(1): 47-61.

[38] Zhou G, He T, Krishnamurthy S, Stankovic J A. Impact of radio irregularity on wireless sensor networks. In Proc. the 2nd ACM MobiSys, June 2004.

[39] Zhu H, Wang J. Chunk-based resource allocation in OFDMA systems-part II: Joint chunk, power and bit allocation. IEEE Transactions on Communications, 2012, 60(2): 499-509.
[40] Takaishi D, Nishiyama H, Kato N, Miura R. Toward energy efficient big data gathering in densely distributed sensor networks. IEEE Transactions on Emerging Topics in Computing, 2014, 2(3): 388-397.

[41] Zeng X, Bagrodia R, Gerla M. Glo-MoSim: A library for parallel simulation of large-scale wireless networks. In Proc. the 12th IEEE PADS, May 1998, pp.154-161.

[42] Shnayder V, Hempstead M, Chen B, Werner-Allen G, Welsh M. Simulating the power consumption of large-scale sensor network applications. In Proc. the 2nd ACM SenSys, Nov. 2004, pp.188-200.

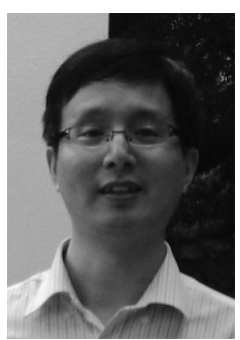

Hai-Ming Chen is an assistant professor at the Institute of Computing Technology, Chinese Academy of Sciences, Beijing. His research areas include wireless, ad hoc, sensor networks, and networked embedded computing systems. He is a member of CCF, ACM, IEEE.

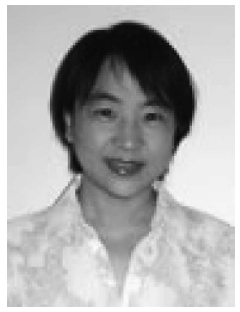

Li Cui is a professor at the Institute of Computing Technology, Chinese Academy of Sciences (CAS), Beijing. She received "The 100 Talents Program of CAS" Award in 2004. Her current research is focused on sensor technology and wireless sensor networks. She is a fellow of the Institution of Engineering and Technology (FIET), a fellow of the Royal Society of Chemistry (FRSC), and a senior member of CCF.

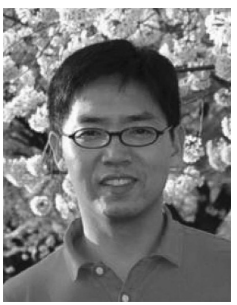

Gang Zhou is an associate professor in the Department of Computer Science at the College of William and Mary, Williamsburg. He received NSF CAREER Award in 2013. His research interests include wireless networks, sensor networks, ubiquitous computing, and mobile computing. He is a senior member of ACM and also a senior member of IEEE. 